\title{
Identification of therapeutic targets in chordoma through comprehensive genomic and transcriptomic analyses
}

\author{
Winnie S. Liang, ${ }^{1}$ Christopher Dardis, ${ }^{2}$ Adrienne Helland, ${ }^{1}$ Shobana Sekar, ${ }^{1}$ \\ Jonathan Adkins, ${ }^{1}$ Lori Cuyugan, ${ }^{1}$ Daniel Enriquez, ${ }^{1}$ Sara Byron, ${ }^{1}$ \\ and Andrew S. Little ${ }^{3}$ \\ ${ }^{1}$ Integrated Cancer Genomics Division, Translational Genomics Research Institute, Phoenix, Arizona 85004, \\ USA; ${ }^{2}$ Division of Neurology, Barrow Neurological Institute, St. Joseph's Hospital and Medical Center, \\ Phoenix, Arizona 85013, USA; ${ }^{3}$ Department of Neurosurgery, Barrow Neurological Institute, St. Joseph's \\ Hospital and Medical Center, Phoenix, Arizona 85013, USA
}

Corresponding author: wliang@tgen.org

(C) 2018 Liang et al. This article is distributed under the terms of the Creative Commons Attribution-NonCommercial License, which permits reuse and redistribution, except for commercial purposes, provided that the original author and source are credited.

Ontology terms: neoplasm of the skeletal system

Published by Cold Spring Harbor Laboratory Press

doi: $10.1101 / \mathrm{mcs} . a 003418$
Abstract Chordoma is a rare, orphan cancer arising from embryonal precursors of bone. Surgery and radiotherapy (RT) provide excellent local control, often at the price of significant morbidity because of the structures involved and the need for relatively high doses of RT; however, recurrence remains high. Although our understanding of the genetic changes that occur in chordoma is evolving rapidly, this knowledge has yet to translate into treatments. We performed comprehensive DNA (paired tumor/normal whole-exome and shallow whole-genome) and RNA (tumor whole-transcriptome) next-generation sequencing analyses of archival sacral and clivus chordoma specimens. Incorporation of transcriptomic data enabled the identification of gene overexpression and expressed DNA alterations, thus providing additional support for potential therapeutic targets. In three patients, we identified alterations that may be amenable to off-label FDA-approved treatments for other tumor types. These alterations include FGFR1 overexpression (ponatinib, pazopanib) and copy-number duplication of CDK4 (palbociclib) and ERBB3 (gefitinib). In a third patient, germline DNA demonstrated predicted pathogenic changes in CHEK2 and ATM, which may have predisposed the patient to developing chordoma at a young age and may also be associated with potential sensitivity to PARP inhibitors because of homologous recombination repair deficiency. Last, in the fourth patient, a missense mutation in IGF1R was identified, suggesting potential activity for investigational anti-IGF1R strategies. Our findings demonstrate that chordoma patients present with aberrations in overlapping pathways. These results provide support for targeting the IGF1R/FGFR/EGFR and CDK4/6 pathways as treatment strategies for chordoma patients. This study underscores the value of comprehensive genomic and transcriptomic analysis in the development of rational, individualized treatment plans for chordoma.

[Supplemental material is available for this article.]

\section{INTRODUCTION}

Chordoma is a rare, locally aggressive, and invasive cancer arising from the notochord, which is a cartilage-like mesodermal rod. In vertebrates, the notochord is a precursor to the intervertebral disc. Chordoma is considered a malignant tumor of bone and accounts for $1 \%-4 \%$ of such tumors. The incidence is $<1 / 1,000,000$ per year and approximately 22 cases are diagnosed per year in the United States. 
Chordoma may arise anywhere along the cranio-spinal axis. In adults, $50 \%$ of the cases involve the sacro-coccygeal region, 35\% occur at the base of the skull, and 15\% are found elsewhere in the vertebral column (Chugh et al. 2007). Gross total resection is typically limited by the cancer's proximity to critical neurovascular structures. Thus, adjuvant radiotherapy is used routinely. Patients have a median survival of $7.7 \mathrm{yr}$ (Smoll et al. 2013); the survival rate is $70 \%-72 \%$ at $5 \mathrm{yr}$ and falls to $40 \%-48 \%$ at $10 \mathrm{yr}$ (Smoll et al. 2013; Chambers et al. 2014).

The brachyury ( $T$, T-box transcription factor $T$ ) protein is essential for notochord differentiation. Expression of the $T$ gene is used to differentiate chordoma from other chondroid tumors (Vujovic et al. 2006; Pillay et al. 2012). A germline single-nucleotide polymorphism (SNP) within the DNA-binding region of brachyury has been strongly associated with the risk of developing sporadic chordoma, whereas duplication of the T gene has been reported in the rare familial form of chordoma (Yang et al. 2009).

Previous analyses have led to the identification of pathological changes in chordomas. Such changes include transcriptomic changes (Bell et al. 2016), as well as point mutations and copy-number losses in genes such as ALK, CDKN2A, NRAS, PTEN, NBPF1, SETD2, and SMARCB1 (Hallor et al. 2008; Le et al. 2011; Choy et al. 2014; Fischer et al. 2015; Wang et al. 2016; Sa et al. 2017; Cote et al. 2018), whose loss characterizes poorly differentiated chordomas (Mobley et al. 2010). Of note, loss of INI1/SMARCB1 may also represent a discrete entity with a more aggressive phenotype more similar to rhabdoid tumors (Hasselblatt et al. 2016). As a rare and typically fatal condition with a complex etiology, and given the variety of molecular mechanisms that may be responsible for the development of this tumor, chordoma may be a candidate for individualized approaches to molecularly targeted treatment. Thus, in this study, we performed next-generation DNA and RNA sequencing of paired chordoma and germline samples to build on our understanding of the genomic and transcriptomic landscape of chordoma and to identify potentially actionable alterations that may be worth exploring further in clinical trials.

\section{RESULTS}

Across the four chordoma patients (Table 1), a mean target coverage of $165 \times$ was achieved for the tumors and 112x for the germline exomes. From the long-insert whole genomes (LIWGs), a mean clonal coverage of $43 \times$ was achieved for the tumors and $44 \times$ for the germline samples. A mean of 195 million paired reads was generated across the tumor RNAs using RNA-seq (RNA sequencing). The total number of somatic alterations identified in each patient's tumor (loss of function, missense, intronic, in-frame indel, splice site loss, splicing altered, untranslated region [UTR]) were $24,32,2$, and 22, respectively for Patients $1-4$. The brachyury (T) SNP rs2305089 was observed in all the tumors (Pillay et al. 2012) along with elevated expression of $T$ in Patients 1, 2, and 4 with FPKMs of 155.5, 398.2, and 442.9, respectively. Table 2 lists the DNA or RNA alterations that were identified through sequencing

\begin{tabular}{lccllc}
\hline \multicolumn{5}{l}{ Table 1. Summary of patients } \\
\hline Patient & Gender & Age at diagnosis & Ethnicity & Location & Tumor purity (\%) \\
\hline 1 & M & 70 & Caucasian & Sacral & 36 \\
2 & M & 57 & Caucasian & Sacral & 74 \\
3 & M & 29 & Caucasian & Sacral & 33 \\
4 & M & 54 & Caucasian & Petro-clival & 44 \\
\hline
\end{tabular}

Phenotypic information for each patient is shown.

${ }^{a}$ Tumor purities were estimated in silico using Sequenza (Favero et al. 2015). 


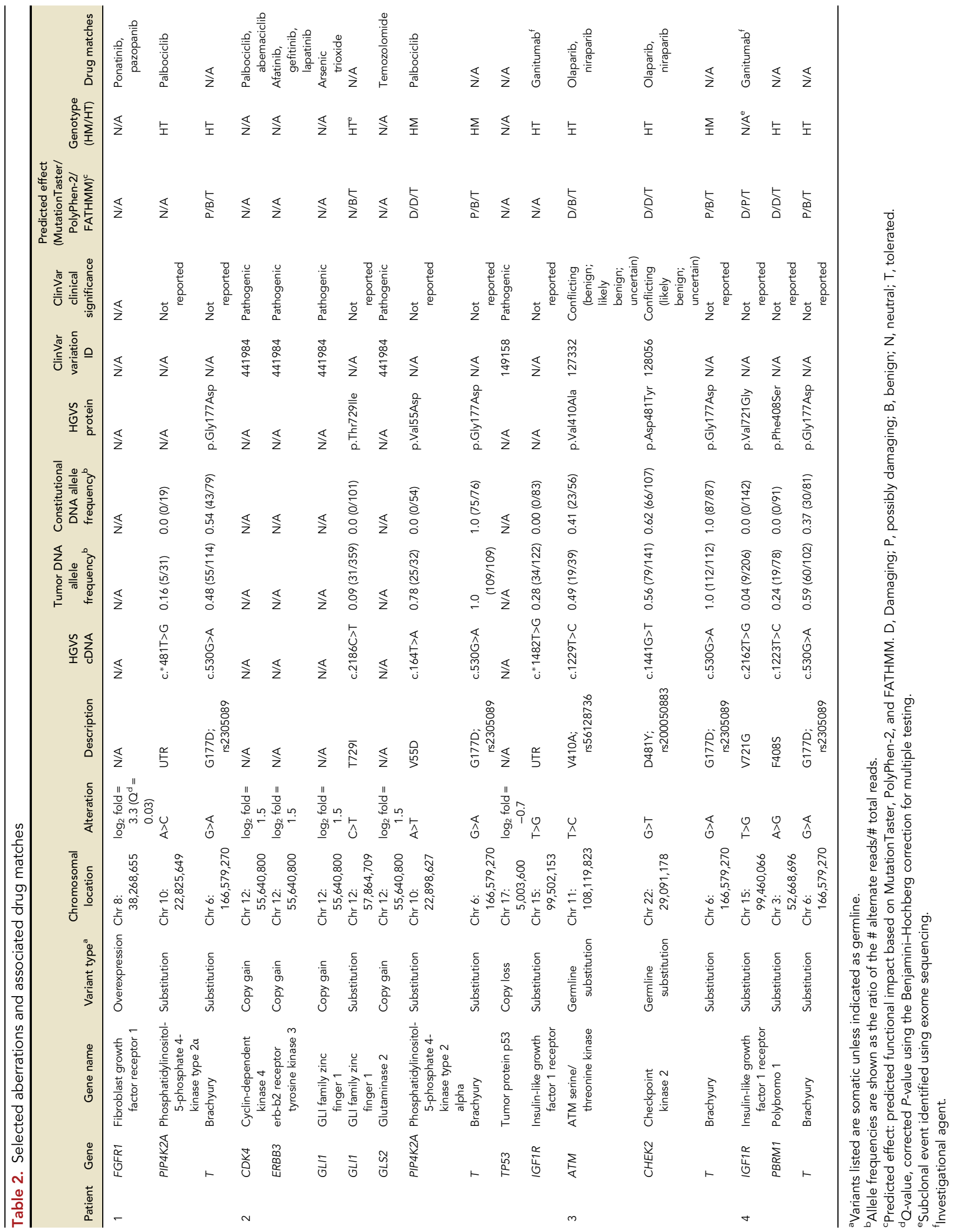


and that are potentially targetable with an FDA-approved agent. For those genes associated with a drug-gene rule, any additional alterations that were detected in those genes across the cohort are also listed. Additional somatic alterations implicated in chordoma or other cancers (ALK, CDKN2A, NRAS, PTEN, SMARCB1, NBPF1, TP3) and potential pathogenic germline variants were also evaluated. Herein, we present clinical information, pathology results, and representative imaging data for each patient, as well as the results from sequencing and drug-gene analyses.

\section{Sacral Chordomas}

\section{Patient 1}

A 70-yr-old male patient presented with a $14 \times 11 \times 20-\mathrm{cm}$ predominantly presacral mass and bony destruction of the distal sacrum and coccyx, as well as multiple amorphous calcifications. Surgery consisted of an S1-S3 laminectomy with en bloc resection of S3 and removal of the distal sacrum and coccyx (Fig. 1, upper row). Pathology reported the tumor to be chondromyxoid, with mild atypia and no mitoses or necrosis, as well as positive immunohistochemistry (IHC) for S100 and keratin AE1/AE3. Eleven months after diagnosis, a magnetic resonance imaging (MRI) showed recurrent local disease (Fig. 1, lower row), with pelvic and inguinal lymphadenopathy and pulmonary metastases. He began palliative treatment with intensity-modulated radiotherapy (IMRT) at that time with $15 \times 2.5=37.5$ Gy to the entire pelvis and an additional $5 \times 2.5=12.5$ Gy to the tumor, along with imatinib (400 mg daily). He developed intercurrent pneumonia and was transferred to hospice care.

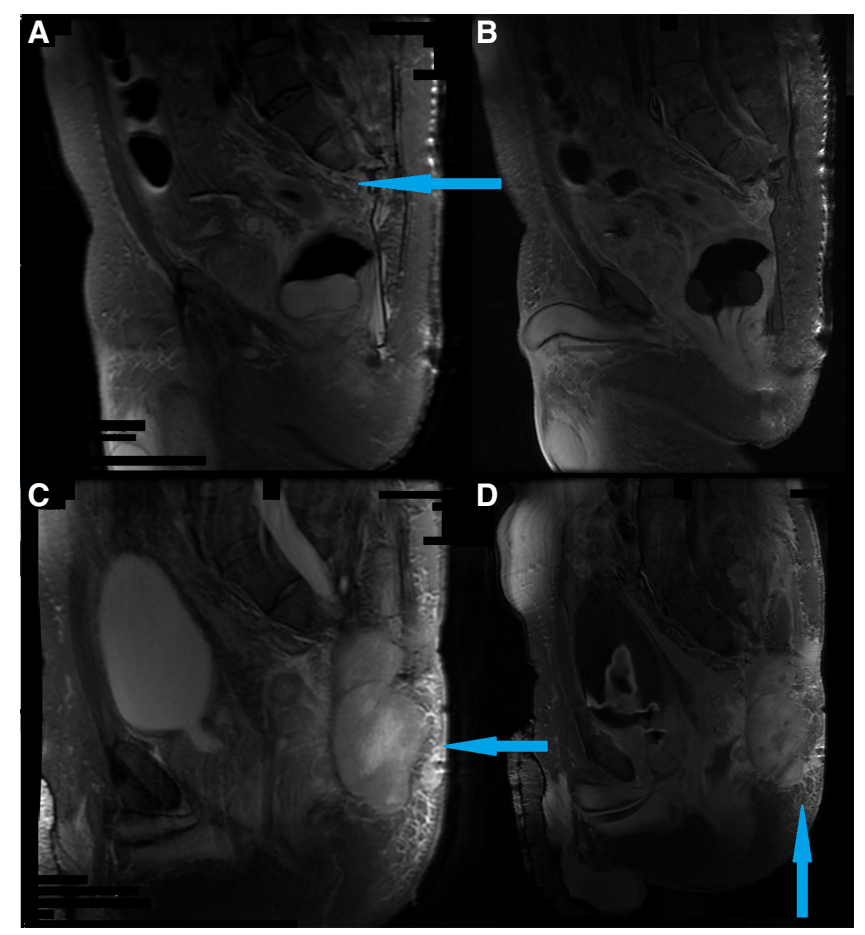

Figure 1. Patient $1 \mathrm{MRI}$ images. All images are sagittal in midline. Panels in the left column $(A, C)$ are T2weighted, fast-spin echo, whereas those in the right column $(B, D)$ are T1-weighted with contrast $(T 1+\mathrm{Gd})$. Panels shown in the upper row $(A, B)$ are postoperative, following initial gross total resection. Resection of the vertebrae caudal to $S 1$ is indicated with the blue arrow. Panels in the lower row $(C, D)$ were taken at time of recurrence. A large, heterogeneous mass extending rostrally almost to S1 and dorsally almost to the skin is present (blue arrows). The dimensions are almost equal to those of the tumor prior to initial resection. 
Tumor DNA sequencing revealed no definitive therapeutic targets. However, a somatic point mutation in the UTR of PIP4K2A was identified and may thus impact transcriptional regulation of the gene. PIP4K2A has been reported to be essential for cell proliferation in acute myeloid leukemia, through AKT/mTOR activation and cell cycle regulation (Jude et al. 2015), and has also been found to be a target of the CDK4/6 inhibitor palbociclib (Sumi et al. 2015). Although PIP4K2A expression was not significantly up-regulated compared to a universal human control, expression of the gene was observed, with a FPKM of 17.8. Clinical trials are currently underway evaluating palbociclib as a single agent in advanced chordoma (NCT03110744), and in CDK4-amplified solid tumors (NCI Match, NCT02465060).

Overexpression of FGFR1 compared to a universal RNA control was also observed in Patient 1's tumor. The FGF pathway has been implicated in chordoma as a driver of brachyury overexpression (Nelson et al. 2012; Hu et al. 2014), which was observed in this patient's tumor. In preclinical models, chordoma cell lines express both FGFR2 and FGFR3 and are sensitive to FGFR inhibition (Hu et al. 2014). FDA-approved agents that target FGFRs include ponatinib, a pan-FGFR inhibitor, and pazopanib, a multityrosine-kinase inhibitor, which also has activity against VEGFR and PDGFR (Schutz et al. 2011; Gozgit et al. 2012). Several other FGFR inhibitors are also in clinical development.

With the availability of RNA-seq data, we further evaluated the expression of factors downstream from FGFR1 to determine if evidence for pathway activation is present. Genes downstream from FGFR demonstrated expression (FPKM >1) with a mean FPKM of 42.7 (see Methods), but none demonstrated differential expression compared to a universal RNA control (corrected $P<0.05$ ). Eight of 22 genes demonstrated higher levels of expression with FPKMs of $>42.7$ and ranging from 46.9 to 147.0. Although these data are not confirmatory of FGFR pathway activation, such data provide evidence that it is possible that pathway activation may have been present in this patient's tumor.

\section{Patient 2}

A 57-yr-old male presented with a $15 \times 14 \times 6$-cm sacral mass. The two-stage surgery included a sacrectomy with $\mathrm{S} 1$ and $\mathrm{S} 2$ interspace embolization followed by closure of a myocutaneous flap. His postoperative MRI is shown in Figure 2. Pathology showed a tumor arranged in cords and lobules within a myxoid background and no mitoses or necrosis. A focus of lymphovascular invasion was identified. Four years after diagnosis, recurrence was noted along the borders of the resection cavity. He enrolled in a Phase 2 clinical trial (NCT02383498) of IMRT (70 Gy) with placebo versus a brachyury vaccine (GI-6301). Three years later, he remained free of further progression and continued to use the vaccine every $3 \mathrm{mo}$, according to the trial protocol.

Sequencing revealed somatic DNA alterations clustered within regions of Chromosome 12 that are associated with targets for FDA-approved drugs, such as CDK4 and ERBB3. For instance, CDK4 amplification commonly occurs in liposarcomas, and the CDK4/6 inhibitor palbociclib showed activity in a Phase 2 trial for advanced liposarcoma (Dickson et al. 2013). In chordoma cell lines lacking p16, likely as a result of CDKN2A loss, CDK4/6 gene expression was observed to suggest the possibility of activation in this pathway (von Witzleben et al. 2015). Subsequent treatment of these lines with the CDK4/6 inhibitors palbociclib and abemaciclib inhibited cell growth to demonstrate the potential efficacy of this treatment strategy. In addition, overexpression of ERBB3 correlated with gefitinib sensitivity in a preclinical lung cancer model (von Witzleben et al. 2015) to suggest that ERBB inhibitors or monoclonal antibodies may have activity in this context. Although the expression levels of CDK4 and ERBB3 were not significantly altered compared to a universal RNA reference $(P>$ 0.05), both genes demonstrated expression with FPKMs of 80.5 and 76.3, respectively. Furthermore, although variants associated with CDKN2A loss were not observed in the DNA, CDKN2A expression was low, with a FPKM of 3.5. 


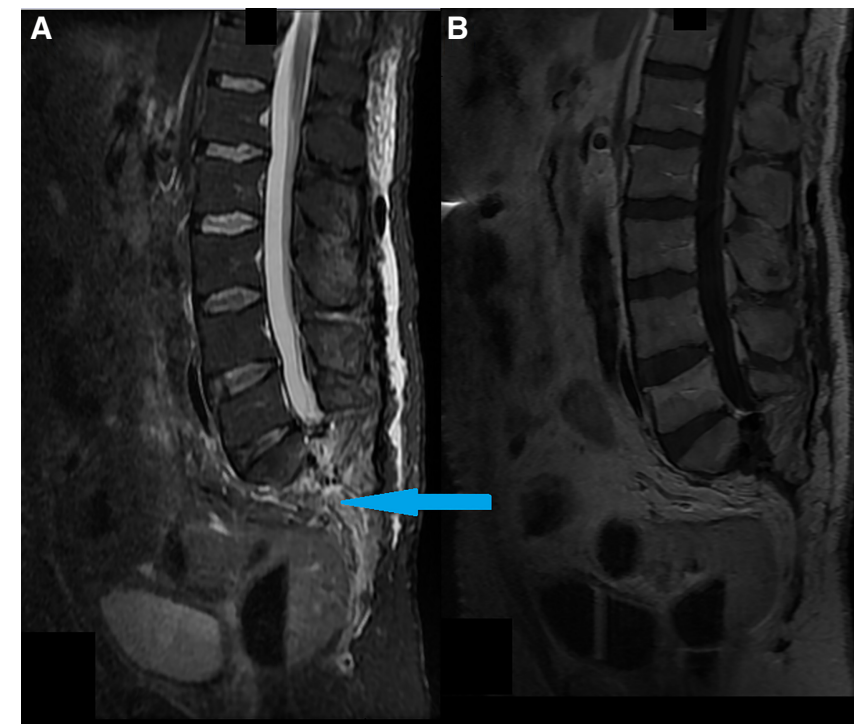

Figure 2. Patient 2 MRI images. Images for Patient 2 after his initial resection. (A) T2-weighted, with fat saturation via short-tau inversion recovery (STIR). (B) T1 + Gd. As with Patient 1 (Fig. 1A,B), resection of the vertebrae caudal to $\mathrm{S} 1$ is seen (blue arrow).

Sequencing also revealed alterations in GLI1 and GLS2, which encode candidate drug targets. For instance, arsenic trioxide binds GLI1 to inhibit transcription and cell growth in a cell culture model of Ewing sarcoma (Beauchamp et al. 2011). In Patient 2's tumor, a missense mutation in GLI1 was detected at a low DNA allele frequency $(0.08)$, but there were no changes in the RNA expression. Furthermore, GLS2 overexpression is associated with decreased MGMT expression and increased sensitivity to temozolomide in a cell culture model of glioblastoma (Szeliga et al. 2012). In Patient 2's tumor, the GLS2 copy number was increased but there were no changes in gene expression. Unlike CDK4 and ERBB3, GLI1 and GLS2 demonstrated low expression, with FPKMs of 1.6 and 2.7, respectively.

Other potentially pathogenic somatic abnormalities were observed, although these do not suggest a specific treatment strategy. An expressed missense mutation in PIP4K2A was detected (RNA allele frequency $[R A F]=0.28)$, along with low-level loss of TP53 $\left(\log _{2}=\right.$ -0.7). This specific PIP4K2A mutation has not been previously reported or characterized, and the functional consequence is unclear. However, as discussed above, PIP4K2A has been identified as a potential target of palbociclib, raising interest in evaluating the CDK4/6 inhibitor in this setting. A heterozygous IGF1R UTR point mutation was also observed. IGF1R has been implicated in chordoma, as $40 \%$ of chordomas demonstrate IGF1R/IR signaling activation and IGF1R is correlated with chordoma tumor volume (Sommer et al. 2010; Scheipl et al. 2012). Additionally, UTR variants have been proposed to regulate IGF1R expression. In this tumor, elevated expression of IGF1R was observed with an FPKM of 45.7, although the gene was not significantly overexpressed compared to a universal RNA reference $(P>$ $0.05)$. The investigational IGF1R antibody, ganitumab, is currently being evaluated in clinical trials for other tumor types.

\section{Patient 3}

An incidental presacral mass was identified in a 29-yr-old male who had experienced chronic tailbone pain since age 18 . The $4.5 \times 8.5 \times 4.5-\mathrm{cm}$ tumor was removed en bloc (Fig. 3), and the chronic pain resolved. Pathology showed physaliferous cells arranged in cords, sheets, 


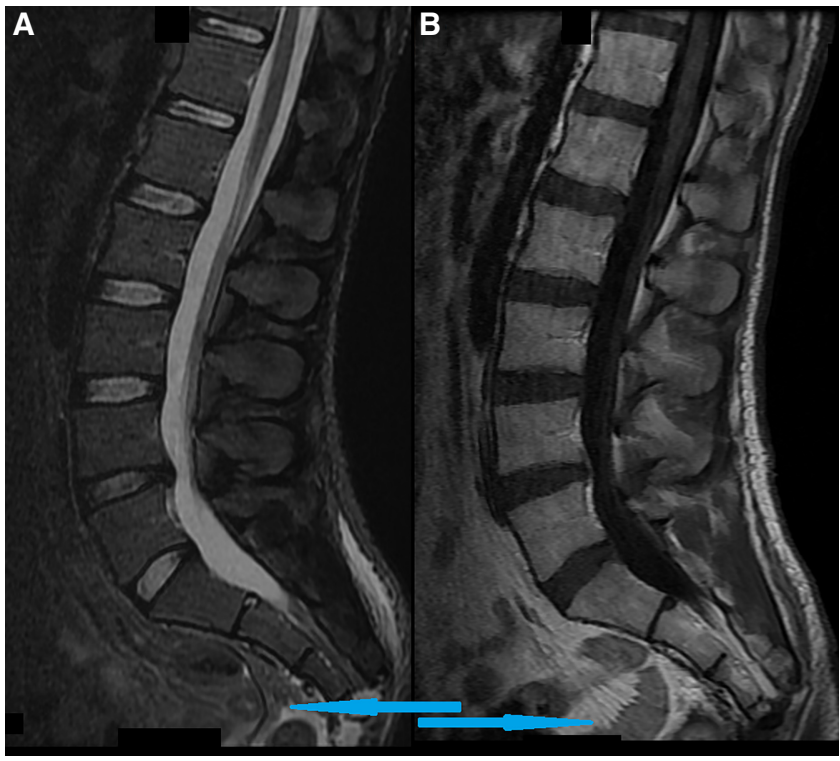

Figure 3. Patient 3's MRI images. Postoperative images for Patient 3. (A) T2-weighted, fat saturated (STIR). (B) T1 + Gd. These images are largely normal; signs of resection of the presacral mass are present (blue arrows).

and nests, forming cyst-like masses separated by fibrocollagenous septa, within a myxoid background. Mild to moderate atypia was noted. IHC for S100, keratin AE1/AE3, and EMA confirmed the diagnosis. Focal invasion of the resection margins was noted. Proton beam radiotherapy was completed, $1.8 \times 42=75.6 \mathrm{~Gy}$, and $8 \mathrm{yr}$ following resection, there were no signs of progression clinically or on MRI.

No somatic DNA or RNA alterations were found to be associated with the targets for FDA-approved drugs. However, evaluation of germline data led to the identification of rare missense mutations in CHEK2 (D481Y; population allele frequency $[\mathrm{PAF}]=0.001$ ) and ATM (V410A; PAF $=0.004)$. These genes encode proteins involved in DNA damage response and thus may have predisposed the patient to cancer. The same germline ATM mutation has been described in breast and ovarian cancer, as well as in acute lymphoblastic leukemia (Thorstenson et al. 2003; Liberzon et al. 2004; Broeks et al. 2008). The CHEK2 mutation we identified is located in the protein kinase domain. Although this mutation has not been reported, similar CHEK2 germline mutations have been reported in Li-Fraumeni syndrome (Bell et al. 1999) and breast cancer (Allinen et al. 2001; Domagala et al. 2012). Both mutations were found in the tumor, although loss of the other allele was not detected in the tumor for either gene. The overall expression of CHEK2 was low in the tumor (FPKM = 3.6), whereas ATM expression was elevated (FPKM = 52.4). Clinical trials are underway evaluating PARP inhibitors (olaparib, niraparib, talazoparib) in tumors with homologous recombination deficiency, including tumors with ATM or CHEK2 loss (e.g., TAPUR, NCT02693535; NCT02286687; NCT03375307). Inhibition of ATM is also being explored and such treatment has been shown to radiosensitize glioma cells (Golding et al. 2009). The patient's younger age supports the hypothesis that these mutations may be pathogenic (Chambers et al. 2014).

\section{Chordoma of Clivus}

Patient 4

A 54-yr-old male was diagnosed with a petro-clival mass and significant brainstem compression. Three-stage resection (Fig. 4, after Stage 1) was followed by adjuvant Cyber Knife 


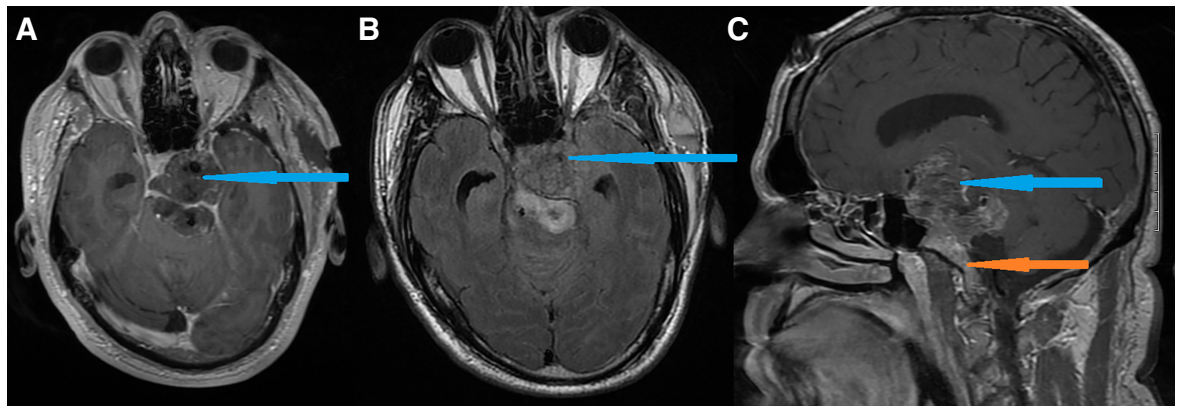

Figure 4. Patient 4's MRI images. Images recorded following the first stage of resection for Patient 4 . Both panels shown are axial. (A) Tw-weighted (FLAIR, fluid-attenuated inversion recovery); $(B) T 1+G d$; $(C)$ sagittal $\mathrm{T} 1+\mathrm{Gd}$. Although the majority of the tumor has been resected (blue arrows), a significant component remains (orange arrow), arising from the clivus.

radiosurgery to remove residual tumor. Pathology showed sheets of cells (physaliferous or epithelioid) within a chondromyxoid background. As with Patient 3, IHC for S100, keratin AE1/AE3, and EMA was confirmatory. The MIB-1 labeling index was $1 \%$, and no mitoses were seen. The patient remains free of progression 18 mo after initial diagnosis.

Exome sequencing revealed a subclonal missense IGF1RV721G mutation that was identified in $4 \%$ of reads. Expression of the mutation-containing transcript was also observed, with $11 \%$ of RNA reads supporting this event. A case study reported that an adult spinal chordoma patient who was treated with an investigational IGF1R/IR inhibitor linsitinib, in addition to the EGFR inhibitor erlotinib, remained stable on treatment for $5 \mathrm{yr}$ (Aleksic et al. 2016). In a separate evaluation of dose escalation of linsitinib with erlotinib in solid tumors, a spinal chordoma patient also demonstrated a partial response to this combination (Macaulay et al. 2016). In Patient 4's tumor, both EGF and EGFR were expressed (FPKM $=50.9$ and 70.0, respectively). Anti-IGF1R strategies continue to be explored in clinical trials.

A missense mutation in PBRM1 (F408S) was also expressed in the tumor (DNA allele frequency $=0.24, \mathrm{RAF}=0.40$ ). $P B R M 1$ encodes a component of complexes required for transcriptional activation via nuclear hormone receptors. Mutations in this gene are associated with renal cell carcinoma (Wang et al. 2017; de Velasco et al. 2018; Ricketts et al. 2018). In a targeted sequencing study, both deletion and missense mutation (S1315F) of PBRM1 have been reported in chordomas (Wang et al. 2016).

\section{Pathway Analysis}

Pathway analysis on differentially expressed genes (corrected $P<0.05$ ) was additionally performed for each patient's tumor (Supplemental Table S1; Patient $1=482$ genes, Patient $2=$ 615 genes, Patient $3=561$ genes, Patient $4=482$ genes). Although analysis did not yield significant $(P<0.05)$ pathways that are specific to drug-gene matches for each patient, potentially interesting alterations were observed. In Patients 1 and 2, a potential immune component was revealed with the identification of $\mathrm{T}$ - and B-cell receptor pathways, and from a cohort-level analysis of all tumors, we observed additional commonly impacted processes across all tumors. These processes include ILK (integrin-linked kinase), integrin, and GTPase signaling, which are associated with the PI3K/Akt/mTor pathways; phospholipase $\mathrm{C}$ and calcium signaling pathways, which are associated with Fgf signaling; regulation of gene expression through the nuclear receptors FXR (farnesoid $X$ receptor), TR (thyroid hormone receptor), LXR (liver $X$ receptor), and RXR (retinoid X receptor); and what appear to be tumor microenvironment processes as defined by epithelial adherens junction signaling and tight junction signaling. Although ILK signaling has not been specifically reported in 
chordomas, obstructing $\beta 1$ integrin function was suggested to be therapeutically relevant following radiation in chordomas (Harryman et al. 2017). Separately, the importance of the microenvironment of chordomas has been previously demonstrated through in vitro analyses, whereby hypoxia combined with exposure to CCN2, a connective tissue growth factor, up-regulated notochord progenitor markers (Patel et al. 2014). Although limited conclusions may be drawn from expression data alone from this small cohort, these findings shed additional light into the intricacies of chordomas.

\section{DISCUSSION}

Our work demonstrates that integrated and comprehensive DNA and RNA profiling is practical on archival chordoma samples and that such an approach aids in the identification of potential candidate therapeutic targets. Previous genetic studies of chordoma have reported somatic abnormalities in genes such as CDKN2A, PTEN, PIK3CA, and TP53 (Hallor et al. 2008; Choy et al. 2014; Fischer et al. 2015; Wang et al. 2016). Similar to previous studies, we did not observe highly recurrent somatic alterations; however, we identified alterations in genes that have been previously associated with chordoma, including PBRM1 (Wang et al. 2016) and IGF1R (Sommer et al. 2010; Scheipl et al. 2012). Incorporation of RNA-seq data was also beneficial, as it clarified which DNA alterations are expressed, and thus may serve as therapeutic targets. Based on comprehensive analyses, potential therapeutic strategies were identified across all patients. In the third patient, we identified germline base substitutions in CHEK2 and ATM, which are likely key driving events in this tumor. With the availability of RNA-seq data, we additionally performed pathway analyses and identified processes that may be associated with potentially targetable pathways, although further studies are needed to determine if and how such processes may impact the efficacy of different treatments.

Overall, the data from this study, along with the results of previously published chordoma studies, provide evidence of aberrations converging on overlapping pathways. Although chordoma appears to be a genetically heterogeneous tumor, activation of common pathways may represent key exclusive events in this disease. These include the Ras-Raf-Mek and mTor pathways that lie downstream from IGF1R, EGFR, and FGFR signaling (Patients 1,2 , and 4), as well as cell cycle progression pathways that lie downstream from TP53, which is activated by CHEK2 and ATM (Patient 3) and CDK4/6 (Patient 2). Inhibition of IGF1R, EGFR, FGFR, and CDK4/6 thus represents possible treatment themes for chordoma (Presneau et al. 2009; Sommer et al. 2010). Targeting of the mTor pathway has also been suggested as a potential therapeutic strategy for chordoma (Presneau et al. 2009).

Although we did not detect EGFR amplification or overexpression in the tumors analyzed here, targeting EGFR using erlotinib has shown promising results in two chordoma patients (Singhal et al. 2009; Houessinon et al. 2015), one of whom was refractory to imatinib. Imatinib inhibited tumor growth in a patient-derived chordoma xenograft (Siu et al. 2013), and in a separate Phase 2 study, imatinib inhibited tumor growth in previously progressive patients expressing PDGFB/PDGFRB (Stacchiotti et al. 2012; Hindi et al. 2015); both of these markers were expressed in the patients analyzed here but did not demonstrate significant overexpression in the tumors compared to a universal RNA control. Notably, the EGFR inhibitor afatinib is currently being evaluated in a Phase 2 trial for chordoma patients with EGFRexpressing tumors (NCT03083678) and also appears to be active in the central nervous system in non-small-cell lung carcinoma patients (Kelly et al. 2018). Last, for Patient 3, who appears to have germline disease, potential strategies for consideration include PARP inhibition and ATM inhibition combined with radiation, though ATM inhibitors are not yet FDA-approved. 
Although our study sheds further light onto chordoma and potential treatment options, a number of limitations are apparent. The first is our small sample size, which is directly related to the rare nature of the malignancy and the availability of archival specimens. Second, given the diversity of mutations previously reported in chordoma and those reported here, additional analyses are needed to understand the role of identified mutations in altering functional pathways and how such perturbed pathways enable tumor development and progression in chordoma. Additional studies are also needed to evaluate efficacy and prioritization of candidate single-agent strategies and to explore potential use of combination therapies in this disease.

Even so, despite the rarity of chordoma, previous studies and our analysis here shed valuable light on our understanding of the disease. As the majority of previous chordoma sequencing studies have used targeted sequencing approaches that lack integrated transcriptomic analyses, we present analysis from a unique data set that integrates whole-genome, whole-exome, and whole-transcriptome data across patients. This analysis is thus the first to integrate both DNA and RNA sequencing data in sacral and clivus chordomas and also provides evidence of convergence of alterations in specific pathways. Notably, current clinical trials that are open to chordoma patients are focused on targeting brachyury (NCT02383498), PD-1/PD-L1 (NCT03173950, NCT02989636), SMARCB1/INI-1 (NCT02601950), and CDK4/6 (NCT03110744). Our analyses lend further credence to CDK4/6 inhibition and suggest that targeting IGF1R/EGFR/FGFR signaling may be a new candidate therapeutic strategy. Such convergence and continued molecular characterization of chordomas has the potential to improve our ability to find effective treatment strategies for patients.

\section{METHODS}

\section{Clinical Details}

De-identified clinical histories, pathology reports, and MRI results were reviewed for each patient. For patients who received care at another institution, some of the records were incomplete.

\section{Sample Collection and Extraction}

Chordomas, embedded in optimal cutting temperature compound, and matched whole blood were retrieved from the Biobank Core Facility of the Barrow Neurological Institute at St. Joseph's Hospital and Medical Center. The tissue was homogenized using the Bullet Blender (Next Advance, Inc.) and stainless-steel beads. The lysate was further homogenized using the QIAshredder (QIAGEN) and digested with proteinase K. For nucleic acid purification, we used the AllPrep DNA/RNA Mini Kit (QIAGEN), which allows for the simultaneous purification of genomic DNA and total RNA from the same biological sample. Germline DNA was extracted from whole blood using the Gentra Puregene Blood Kit, 3rd edition (QIAGEN), following the manufacturer's instructions, including RNase A treatment.

\section{Next-Generation Sequencing}

Paired tumor/normal exomes were constructed using SureSelectXT Human All Exon V5+UTR baits (Agilent). The KAPA Library Preparation Kit (Kapa Biosystems) was used for Patient 1 and the KAPA HyperPrep Kit (Kapa Biosystems) was used for Patients 2-4. Paired tumor/normal LIWGs were constructed using the KAPA HyperPrep Kit, as previously described (Liang et al. 2014, 2017). Tumor RNA was used to generate RNA libraries using the TruSeq RNA Access Library Prep Kit (Illumina). The libraries were paired-end sequenced 
using the TruSeq PE Cluster Kit v3 and the TruSeq SBS Kit v3 (Illumina) on the HiSeq 2500 (Illumina) for $2 \times 109$ bp reads with a 7-base index read.

\section{Data Analysis}

Binary base call files were converted to FASTQ files using bcl2fastq Conversion Software (Illumina) v1.8-2.17.1.14. The FASTQ files were aligned to the human reference genome (GRCh37) using the Burrows-Wheeler Aligner (Li and Durbin 2009). Somatic analyses were performed as previously described using an annotation pipeline built on GENCODE 3 (Ensembl; GRCh37.1) (Liang et al. 2017; Zerbino et al. 2018). To determine whether tumor single-nucleotide variants (SNVs) were present, we used the consensus of at least two of the three following tools: Seurat, a Bayesian framework (Christoforides et al. 2013) (threshold quality score of $>30$ ), MuTect, a Bayesian classifier (Cibulskis et al. 2013), and Strelka, a somatic small-variant tool (Saunders et al. 2012). Tumor cellularities, or purities, for each sample were estimated in silico using Sequenza (Favero et al. 2015). MutationTaster (Schwarz et al. 2010), PolyPhen-2 (Adzhubei et al. 2010), and FATHMM (Shihab et al. 2013) were used to predict the impact of identified base substitutions.

DNA copy number and breakpoint detection were assessed using LIWG data (Liang et al. 2014). For copy-number variation (CNV) detection, (1) read depths were determined every 100 bases across sequenced regions; (2) normalized $\log _{2}$ fold changes between the tumor and germline were calculated; and (3) a smoothing window was applied. The circular binary segmentation algorithm was used to partition chromosomes into sections of equal copy number to correct for noise in order to calculate $\log _{2}$ copy fold changes (Olshen et al. 2004).

RNA reads were aligned to GRCh37 using STAR (Dobin et al. 2013). Differential expression analysis was performed for each tumor sample against Universal Human Reference RNA (Agilent) using Cufflinks/Cuffdiff (Trapnell et al. 2012), which performs transcript assembly to identify differentially expressed genes, and DESeq2 (Anders and Huber 2010; Love et al. 2014), which uses the negative binomial distribution. False discovery rate adjustment of $P$ values for expression changes were performed to calculate a $Q$-value, or a corrected $P$-value, using the Benjamini-Hochberg correction for multiple testing. Expression levels are expressed as fragments per kilobase of transcript per million mapped reads (FPKMs). We defined a threshold FPKM value of $>1$ to indicate expression of a gene.

Pathway analysis was performed using differentially expressed genes (corrected $P<0.05$ ) identified using both Cuffdiff and DESeq2. Analysis was performed using the Ingenuity Pathway Analysis (IPA) tool (version 01-12; QIAGEN) to identify potentially perturbed pathways in each tumor. Significant pathways were identified with $P<0.05$. In a separate analysis for Patient 1, KEGG networks N00019 (FGF-FGFR-RAS-ERK signaling pathway) and N00037 (FGF-FGFR-PI3K signaling pathway) were used to identify factors downstream from FGFR; interrogated genes include GRB2, SOS1, SOS2, HRAS, KRAS, NRAS, ARAF, BRAF, RAF1, MAP2K1, MAP2K2, MAPK1, MAPK3, PIK3CA, PIK3CB, PIK3CD, AKT1, AKT2, AKT3, $M T O R, R P S 6 K B 1$, and RPS6KB2.

\section{Drug-Gene Matching}

All somatic aberrations identified through LIWG, exome, and RNA sequencing data, including SNVs, small indels (insertion/deletions), CNVs, translocations, gene fusions, and RNA expression changes, were evaluated for each case. Potential therapeutic implications for RNA expression events are limited to genes that are differentially expressed compared to the Universal Human Reference RNA (corrected $P<0.05$ ). For any abnormalities identified, we looked for potential therapies using a proprietary drug-gene database annotated with a pharmacopeia of FDA-approved agents. Genes and alterations associated with FDA-approved agents are reported here. 
Competing Interest Statement The authors have declared no competing interest.

\section{Referees}

Robert Maki

Anonymous

Received July 30, 2018; accepted in revised form October 4, 2018.

\section{ADDITIONAL INFORMATION}

\section{Data Deposition and Access}

The sequencing data generated from this study are available in the NCBI (National Center for Biotechnology Information) dbGaP (Database of Genotypes and Phenotypes) repository (https://www.ncbi.nlm.nih.gov/gap) under controlled access (accession number phs001643).

\section{Ethics Statement}

This work was approved by the IRB of St. Joseph's Hospital and Medical Center in Phoenix, Arizona. The clinical review was performed on de-identified records. Written informed consent was obtained from the patients to publish the work detailed here.

\section{Acknowledgments}

We would like to thank Jennifer Eschbacher, MD, and Catherine Seiler, PhD, of the Biobank Core Facility (St. Joseph's Hospital and Barrow Neurological Institute); Jessica Aldrich, Dr. Jonathan Keats, and Tyler Izatt for bioinformatics support (TGen): Dr. Michael Berens for support; and Cynthia Lechuga, Diana Davis, and Stephanie Buchholtz (TGen) for administrative support. Nancy Linford, PhD, provided editing assistance.

\section{Author Contributions}

W.S.L. and A.S.L. conceived the study. W.S.L. and C.D. wrote the manuscript. A.H., J.A., and L.C. performed nucleic acid extractions, prepared sequencing libraries, and performed sequencing. S.S. and D.E. performed bioinformatics analyses. S.B. performed drug-gene matching. S.B. and W.S.L. performed data interpretation. All authors reviewed the manuscript.

\section{Funding}

This work was supported by the Barrow Neurological Foundation and the Translational Genomics Research Institute. The funding bodies had no role in the study design, data collection and analysis, decision to publish, or preparation of the manuscript.

\section{REFERENCES}

Adzhubei IA, Schmidt S, Peshkin L, Ramensky VE, Gerasimova A, Bork P, Kondrashov AS, Sunyaev SR. 2010. A method and server for predicting damaging missense mutations. Nat Methods 7: 248-249.

Aleksic T, Browning L, Woodward M, Phillips R, Page S, Henderson S, Athanasou N, Ansorge O, Whitwell D, Pratap S, et al. 2016. Durable response of spinal chordoma to combined inhibition of IGF-1R and EGFR. Front Oncol 6: 98.

Allinen M, Huusko P, Mäntyniemi S, Launonen V, Winqvist R. 2001. Mutation analysis of the CHK2 gene in families with hereditary breast cancer. Br J Cancer 85: 209-212.

Anders S, Huber W. 2010. Differential expression analysis for sequence count data. Genome Biol 11: R106.

Beauchamp EM, Ringer L, Bulut G, Sajwan KP, Hall MD, Lee Y-C, Peaceman D, Özdemirli M, Rodriguez O, Macdonald TJ, et al. 2011. Arsenic trioxide inhibits human cancer cell growth and tumor development in mice by blocking Hedgehog/GLI pathway. J Clin Invest 121: 148-160.

Bell DW, Varley JM, Szydlo TE, Kang DH, Wahrer DC, Shannon KE, Lubratovich M, Verselis SJ, Isselbacher KJ, Fraumeni JF, et al. 1999. Heterozygous germ line hCHK2 mutations in Li-Fraumeni syndrome. Science 286: 2528-2531.

Bell D, Raza SM, Bell AH, Fuller GN, DeMonte F. 2016. Whole-transcriptome analysis of chordoma of the skull base. Virchows Arch 469: 439-449. 
Broeks A, Braaf LM, Huseinovic A, Schmidt MK, Russell NS, van Leeuwen FE, Hogervorst FB, Van't Veer LJ. 2008. The spectrum of ATM missense variants and their contribution to contralateral breast cancer. Breast Cancer Res Treat 107: 243-248.

Chambers KJ, Lin DT, Meier J, Remenschneider A, Herr M, Gray ST. 2014. Incidence and survival patterns of cranial chordoma in the United States. Laryngoscope 124: 1097-1102.

Choy E, MacConaill LE, Cote GM, Le LP, Shen JK, Nielsen GP, lafrate AJ, Garraway LA, Hornicek FJ, Duan Z. 2014. Genotyping cancer-associated genes in chordoma identifies mutations in oncogenes and areas of chromosomal loss involving CDKN2A, PTEN, and SMARCB1. PLoS One 9: e101283.

Christoforides A, Carpten JD, Weiss GJ, Demeure MJ, Von Hoff DD, Craig DW. 2013. Identification of somatic mutations in cancer through Bayesian-based analysis of sequenced genome pairs. BMC Genomics 14: 302.

Chugh R, Tawbi H, Lucas DR, Biermann JS, Schuetze SM, Baker LH. 2007. Chordoma: the nonsarcoma primary bone tumor. Oncologist 12: 1344-1350.

Cibulskis K, Lawrence MS, Carter SL, Sivachenko A, Jaffe D, Sougnez C, Gabriel S, Meyerson M, Lander ES, Getz G. 2013. Sensitive detection of somatic point mutations in impure and heterogeneous cancer samples. Nat Biotechnol 31: 213-219.

Cote GM, He J, Choy E. 2018. Next-generation sequencing for patients with sarcoma: a single center experience. Oncologist 23: 234-242.

de Velasco G, Wankowicz SA, Madison R, Ali SM, Norton C, Duquette A, Ross JS, Bossé D, Lalani AA, Miller VA, et al. 2018. Targeted genomic landscape of metastases compared to primary tumours in clear cell metastatic renal cell carcinoma. Br J Cancer 118: 1238-1242.

Dickson MA, Tap WD, Keohan ML, D'Angelo SP, Gounder MM, Antonescu CR, Landa J, Qin LX, Rathbone DD, Condy MM, et al. 2013. Phase II trial of the CDK4 inhibitor PD0332991 in patients with advanced CDK4amplified well-differentiated or dedifferentiated liposarcoma. J Clin Oncol 31: 2024-2028.

Dobin A, Davis CA, Schlesinger F, Drenkow J, Zaleski C, Jha S, Batut P, Chaisson M, Gingeras TR. 2013. STAR: ultrafast universal RNA-seq aligner. Bioinformatics 29: 15-21.

Domagala P, Wokolorczyk D, Cybulski C, Huzarski T, Lubinski J, Domagala W. 2012. Different CHEK2 germline mutations are associated with distinct immunophenotypic molecular subtypes of breast cancer. Breast Cancer Res Treat 132: 937-945.

Favero F, Joshi T, Marquard AM, Birkbak NJ, Krzystanek M, Li Q, Szallasi Z, Eklund AC. 2015. Sequenza: allelespecific copy number and mutation profiles from tumor sequencing data. Ann Oncol 26: 64-70.

Fischer C, Scheipl S, Zopf A, Niklas N, Deutsch A, Jorgensen M, Lohberger B, Froehlich EV, Leithner A, Gabriel $C$, et al. 2015. Mutation analysis of nine chordoma specimens by targeted next-generation cancer panel sequencing. J Cancer 6: 984-989.

Golding SE, Rosenberg E, Valerie N, Hussaini I, Frigerio M, Cockcroft XF, Chong WY, Hummersone M, Rigoreau L, Menear KA, et al. 2009. Improved ATM kinase inhibitor KU-60019 radiosensitizes glioma cells, compromises insulin, AKT and ERK prosurvival signaling, and inhibits migration and invasion. Mol Cancer Ther 8: 2894-2902.

Gozgit JM, Wong MJ, Moran L, Wardwell S, Mohemmad QK, Narasimhan NI, Shakespeare WC, Wang F, Clackson T, Rivera VM. 2012. Ponatinib (AP24534), a multitargeted pan-FGFR inhibitor with activity in multiple FGFR-amplified or mutated cancer models. Mol Cancer Ther 11: 690-699.

Hallor KH, Staaf J, Jönsson G, Heidenblad M, Vult von Steyern F, Bauer HC, ljszenga M, Hogendoorn PC, Mandahl N, Szuhai K, et al. 2008. Frequent deletion of the CDKN2A locus in chordoma: analysis of chromosomal imbalances using array comparative genomic hybridisation. $\mathrm{Br} J$ Cancer 98: 434-442.

Harryman WL, Gard JMC, Pond KW, Simpson SJ, Heppner LH, Hernandez-Cortes D, Little AS, Eschbacher JM, Cress AE. 2017. Targeting the cohesive cluster phenotype in chordoma via $\beta 1$ integrin increases ionizing radiation efficacy. Neoplasia 19: 919-927.

Hasselblatt M, Thomas C, Hovestadt V, Schrimpf D, Johann P, Bens S, Oyen F, Peetz-Dienhart S, Crede Y, Wefers A, et al. 2016. Poorly differentiated chordoma with SMARCB1/INI1 loss: a distinct molecular entity with dismal prognosis. Acta Neuropathol 132: 149-151.

Hindi N, Casali PG, Morosi C, Messina A, Palassini E, Pilotti S, Tamborini E, Radaelli S, Gronchi A, Stacchiotti S. 2015. Imatinib in advanced chordoma: a retrospective case series analysis. Eur J Cancer 51: 2609-2614.

Houessinon A, Boone M, Constans JM, Toussaint P, Chauffert B. 2015. Sustained response of a clivus chordoma to erlotinib after imatinib failure. Case Rep Oncol 8: 25-29.

Hu Y, Mintz A, Shah SR, Quinones-Hinojosa A, Hsu W. 2014. The FGFR/MEK/ERK/brachyury pathway is critical for chordoma cell growth and survival. Carcinogenesis 35: 1491-1499.

Jude JG, Spencer GJ, Huang X, Somerville TDD, Jones DR, Divecha N, Somervaille TCP. 2015. A targeted knockdown screen of genes coding for phosphoinositide modulators identifies PIP4K2A as required for acute myeloid leukemia cell proliferation and survival. Oncogene 34: 1253-1262.

Kelly WJ, Shah NJ, Subramaniam DS. 2018. Management of brain metastases in epidermal growth factor receptor mutant non-small-cell lung cancer. Front Oncol 8: 208. 
Le LP, Nielsen GP, Rosenberg AE, Thomas D, Batten JM, Deshpande V, Schwab J, Duan Z, Xavier RJ, Hornicek FJ, et al. 2011. Recurrent chromosomal copy number alterations in sporadic chordomas. PLoS One 6: e18846.

$\mathrm{Li} \mathrm{H}$, Durbin R. 2009. Fast and accurate short read alignment with Burrows-Wheeler transform. Bioinformatics 25: 1754-1760.

Liang WS, Aldrich J, Tembe W, Kurdoglu A, Cherni I, Phillips L, Reiman R, Baker A, Weiss GJ, Carpten JD, et al. 2014. Long insert whole genome sequencing for copy number variant and translocation detection. Nucleic Acids Res 42: e8.

Liang WS, Hendricks W, Kiefer J, Schmidt J, Sekar S, Carpten J, Craig DW, Adkins J, Cuyugan L, Manojlovic Z, et al. 2017. Integrated genomic analyses reveal frequent TERT aberrations in acral melanoma. Genome Res 27: 524-532.

Liberzon E, Avigad S, Stark B, Zilberstein J, Freedman L, Gorfine M, Gavriel H, Cohen IJ, Goshen Y, Yaniv I, et al. 2004. Germ-line ATM gene alterations are associated with susceptibility to sporadic T-cell acute lymphoblastic leukemia in children. Genes Chromosomes Cancer 39: 161-166.

Love MI, HuberW, Anders S. 2014. Moderated estimation of fold change and dispersion for RNA-seq data with DESeq2. Genome Biol 15: 550.

Macaulay VM, Middleton MR, Eckhardt SG, Rudin CM, Juergens RA, Gedrich R, Gogov S, McCarthy S, Poondru S, Stephens AW, et al. 2016. Phase I dose-escalation study of linsitinib (OSI-906) and erlotinib in patients with advanced solid tumors. Clin Cancer Res 22: 2897-2907.

Mobley BC, McKenney JK, Bangs CD, Callahan K, Yeom KW, Schneppenheim R, Hayden MG, Cherry AM, Gokden M, Edwards MS, et al. 2010. Loss of SMARCB1/INI1 expression in poorly differentiated chordomas. Acta Neuropathol 120: 745-753.

Nelson AC, Pillay N, Henderson S, Presneau N, Tirabosco R, Halai D, Berisha F, Flicek P, Stemple DL, Stern CD, et al. 2012. An integrated functional genomics approach identifies the regulatory network directed by brachyury (T) in chordoma. J Pathol 228: 274-285.

Olshen AB, Venkatraman ES, Lucito R, Wigler M. 2004. Circular binary segmentation for the analysis of arraybased DNA copy number data. Biostatistics 5: 557-572.

Patel P, Brooks C, Seneviratne A, Hess DA, Séguin CA. 2014. Investigating microenvironmental regulation of human chordoma cell behaviour. PLoS One 9: e115909.

Pillay N, Plagnol V, Tarpey PS, Lobo SB, Presneau N, Szuhai K, Halai D, Berisha F, Cannon SR, Mead S, et al. 2012. A common single-nucleotide variant in $T$ is strongly associated with chordoma. Nat Genet 44: 1185-1187.

Presneau N, Shalaby A, Idowu B, Gikas P, Cannon SR, Gout I, Diss T, Tirabosco R, Flanagan AM. 2009. Potential therapeutic targets for chordoma: PI3K/AKT/TSC1/TSC2/mTOR pathway. Br J Cancer 100: 1406-1414.

Ricketts CJ, De Cubas AA, Fan H, Smith CC, Lang M, Reznik E, Bowlby R, Gibb EA, Akbani R, Beroukhim R, et al. 2018. The cancer genome atlas comprehensive molecular characterization of renal cell carcinoma. Cell Rep 23: 3698

Sa JK, Lee IH, Hong SD, Kong DS, Nam DH. 2017. Genomic and transcriptomic characterization of skull base chordoma. Oncotarget 8: 1321-1328.

Saunders CT, Wong WS, Swamy S, Becq J, Murray LJ, Cheetham RK. 2012. Strelka: accurate somatic smallvariant calling from sequenced tumor-normal sample pairs. Bioinformatics 28: 1811-1817.

Scheipl S, Froehlich EV, Leithner A, Beham A, Quehenberger F, Mokry M, Stammberger H, Varga PP, Lazáry A, Windhager $R$, et al. 2012. Does insulin-like growth factor 1 receptor (IGF-1R) targeting provide new treatment options for chordomas? A retrospective clinical and immunohistochemical study. Histopathology 60: 999-1003.

Schutz FA, Choueiri TK, Sternberg CN. 2011. Pazopanib: clinical development of a potent anti-angiogenic drug. Crit Rev Oncol Hematol 77: 163-171.

Schwarz JM, Rödelsperger C, Schuelke M, Seelow D. 2010. MutationTaster evaluates disease-causing potential of sequence alterations. Nat Methods 7: 575-576.

Shihab HA, Gough J, Cooper DN, Stenson PD, Barker GL, Edwards KJ, Day IN, Gaunt TR. 2013. Predicting the functional, molecular, and phenotypic consequences of amino acid substitutions using hidden Markov models. Hum Mutat 34: 57-65.

Singhal N, Kotasek D, Parnis FX. 2009. Response to erlotinib in a patient with treatment refractory chordoma. Anticancer Drugs 20: 953-955.

Siu IM, Ruzevick J, Zhao Q, Connis N, Jiao Y, Bettegowda C, Xia X, Burger PC, Hann CL, Gallia GL. 2013. Erlotinib inhibits growth of a patient-derived chordoma xenograft. PLoS One 8: e78895.

Smoll NR, Gautschi OP, Radovanovic I, Schaller K, Weber DC. 2013. Incidence and relative survival of chordomas: the standardized mortality ratio and the impact of chordomas on a population. Cancer 119: 2029-2037.

Sommer J, Itani DM, Homlar KC, Keedy VL, Halpern JL, Holt GE, Schwartz HS, Coffin CM, Kelley MJ, Cates JM. 2010. Methylthioadenosine phosphorylase and activated insulin-like growth factor-1 receptor/insulin receptor: potential therapeutic targets in chordoma. J Pathol 220: 608-617. 
Stacchiotti S, Longhi A, Ferraresi V, Grignani G, Comandone A, Stupp R, Bertuzzi A, Tamborini E, Pilotti S Messina A, et al. 2012. Phase II study of imatinib in advanced chordoma. J Clin Oncol 30: 914-920.

Sumi NJ, Kuenzi BM, Knezevic CE, Remsing Rix LL, Rix U. 2015. Chemoproteomics reveals novel protein and lipid kinase targets of clinical CDK4/6 inhibitors in lung cancer. ACS Chem Biol 10: 2680-2686.

Szeliga M, Zgrzywa A, Obara-Michlewska M, Albrecht J. 2012. Transfection of a human glioblastoma cell line with liver-type glutaminase (LGA) down-regulates the expression of DNA-repair gene MGMT and sensitizes the cells to alkylating agents. J Neurochem 123: 428-436.

Thorstenson YR, Roxas A, Kroiss R, Jenkins MA, Yu KM, Bachrich T, Muhr D, Wayne TL, Chu G, Davis RW, et al. 2003. Contributions of ATM mutations to familial breast and ovarian cancer. Cancer Res 63: 3325-3333.

Trapnell C, Roberts A, Goff L, Pertea G, Kim D, Kelley DR, Pimentel H, Salzberg SL, Rinn JL, Pachter L. 2012. Differential gene and transcript expression analysis of RNA-seq experiments with TopHat and Cufflinks. Nat Protoc 7: 562-578.

von Witzleben A, Goerttler LT, Marienfeld R, Barth H, Lechel A, Mellert K, Böhm M, Kornmann M, MayerSteinacker R, von Baer A, et al. 2015. Preclinical characterization of novel chordoma cell systems and their targeting by pharmocological inhibitors of the CDK4/6 cell-cycle pathway. Cancer Res 75: 3823-3831.

Vujovic S, Henderson S, Presneau N, Odell E, Jacques TS, Tirabosco R, Boshoff C, Flanagan AM. 2006. Brachyury, a crucial regulator of notochordal development, is a novel biomarker for chordomas. $J$ Pathol 209: 157-165.

Wang L, Zehir A, Nafa K, Zhou N, Berger MF, Casanova J, Sadowska J, Lu C, Allis CD, Gounder M, et al. 2016. Genomic aberrations frequently alter chromatin regulatory genes in chordoma. Genes Chromosomes Cancer 55: 591-600.

Wang H, Qu Y, Dai B, Zhu Y, Shi G, Shen Y, Zhang H, Ye D. 2017. PBRM1 regulates proliferation and the cell cycle in renal cell carcinoma through a chemokine/chemokine receptor interaction pathway. PLoS One 12: e0180862.

Yang XR, Ng D, Alcorta DA, Liebsch NJ, Sheridan E, Li S, Goldstein AM, Parry DM, Kelley MJ. 2009. T (brachyury) gene duplication confers major susceptibility to familial chordoma. Nat Genet 41: 1176-1178.

Zerbino DR, Achuthan P, Akanni W, Amode MR, Barrell D, Bhai J, Billis K, Cummins C, Gall A, Giron CG, et al. 2018. Ensembl 2018. Nucleic Acids Res 46: D754-D761. 


\section{COLD SPRING HARBOR Molecular Case Studies}

\section{Identification of therapeutic targets in chordoma through comprehensive genomic and transcriptomic analyses}

Winnie S. Liang, Christopher Dardis, Adrienne Helland, et al.

Cold Spring Harb Mol Case Stud 2018, 4: a003418 originally published online October 15, 2018 Access the most recent version at doi: $10.1101 /$ mcs.a003418
Supplementary http://molecularcasestudies.cshlp.org/content/suppl/2018/11/05/mcs.a003418.D Material C1
References This article cites 65 articles, 11 of which can be accessed free at: http://molecularcasestudies.cshlp.org/content/4/6/a003418.full.html\#ref-list-1
License This article is distributed under the terms of the Creative Commons Attribution-NonCommercial License, which permits reuse and redistribution, except for commercial purposes, provided that the original author and source are credited.
Email Alerting Receive free email alerts when new articles cite this article - sign up in the box at the Service top right corner of the article or click here.

